\title{
Preface: The $X$ in the Latinx Files
}

\begin{abstract}
Although the $X$ in Latinx may be challenging to pronounce, it is doing the important work of fashioning a new inclusive language. It is a marker that the structures of language that have been founded in gender discrimination are being realigned. This new usage of $X$ embraces multiplicity rather than manifesting worn-out binaries and allows for the undefined and unexplained. The $X$ in The Latinx Files, which combines Latinx with The X-Files, expresses a similar dynamic, that Latinx science fiction is rearranging the structures of science fiction that have not done right by Latinx communities. The space alien in particular is able to express the concerns of Latinx communities, not as the Other but as a Multitude. The Latinx Files evokes a fictional television show, in the hopes that Latinx science fiction continues to flourish in print and on the screen, and it demonstrates that space aliens are helping to envision a radically inclusive society.
\end{abstract}





\section{The Latinx Files}


